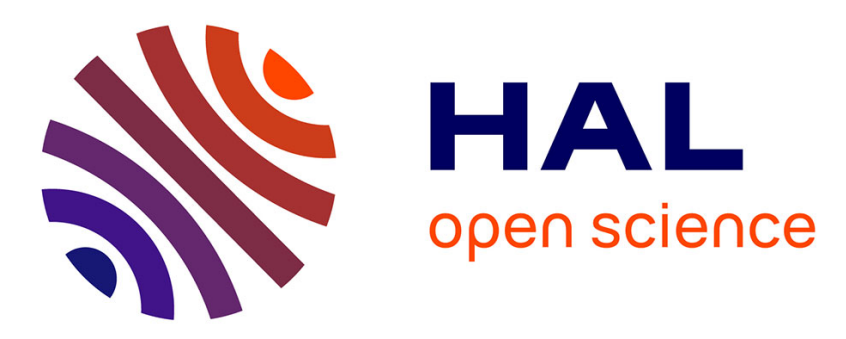

\title{
Uniform Stability Analysis for Time-Varying Systems Applying Homogeneity
}

\author{
Hector Rios, Denis Efimov, Leonid Fridman, Jaime Moreno, Wilfrid \\ Perruquetti
}

\section{- To cite this version:}

Hector Rios, Denis Efimov, Leonid Fridman, Jaime Moreno, Wilfrid Perruquetti. Uniform Stability Analysis for Time-Varying Systems Applying Homogeneity. IEEE CDC 2014, Dec 2014, Los Angeles, United States. hal-01082173

\section{HAL Id: hal-01082173 https://hal.inria.fr/hal-01082173}

Submitted on 13 Nov 2014

HAL is a multi-disciplinary open access archive for the deposit and dissemination of scientific research documents, whether they are published or not. The documents may come from teaching and research institutions in France or abroad, or from public or private research centers.
L'archive ouverte pluridisciplinaire HAL, est destinée au dépôt et à la diffusion de documents scientifiques de niveau recherche, publiés ou non, émanant des établissements d'enseignement et de recherche français ou étrangers, des laboratoires publics ou privés. 


\title{
Uniform Stability Analysis for Time-Varying Systems Applying Homogeneity
}

\author{
Ríos H., Efimov D., Fridman L., Moreno J., Perruquetti W.
}

\begin{abstract}
The uniform stability notion for a class of nonlinear time-varying systems is studied using the homogeneity framework. It is assumed that the system is weighted homogeneous considering the time variable as a constant parameter, then several conditions of uniform stability for such a class of systems are formulated. The results are applied to the problem of adaptive estimation for a linear system.
\end{abstract}

\section{INTRODUCTION}

For homogeneous time-invariant dynamical systems, the global behavior of trajectories can be evaluated based on their behavior on a suitably defined sphere around the origin [1]. Thus the local and global behaviors of homogeneous systems are the same. This property has been found useful for stability analysis [2], [1], [3], [4], [5], approximation of system dynamics [6], [7], stabilization [8], [9], [10], [11], [12] and estimation [2], [7]. It has been shown that for stability/instability analysis, Lyapunov function of a homogeneous system can be chosen homogeneous [13], [5], [14]. Thus the numerical analysis and design of homogeneous systems may be simpler since, for example, a Lyapunov function has to be constructed on a sphere only (on the whole state space it can extended using homogeneity). In addition, the homogeneous systems have certain intrinsic robustness properties [15], [16].

In many cases the system dynamics is perturbed by exogenous disturbances, whose known parts can be modeled by some time functions, then another class of models arise: timevarying dynamical systems. Parameters of these disturbances (the rate of convergence or the main frequency) influence a lot on the system stability. For example, a nonlinear system can be stable for one exponentially converging disturbance and unstable with respect to another one, another example is the resonance phenomenon in linear systems. Due to robustness properties of homogeneous systems it would be

Ríos H., Efimov D. and Perruquetti W. are with Non-A team @ Inria, Parc Scientifique de la Haute Borne, 40 avenue Halley, 59650 Villeneuve d'Ascq, France.

Fridman L. is with Departamento de Control y Robótica, División de Ingeniería Eléctrica, Facultad de Ingeniería, Universidad Nacional Autónoma de México (UNAM), C.P. 04510, México, D.F., Mexico.

Moreno J. is with Eléctrica y Computación, Instituto de Ingeniería, Universidad Nacional Autónoma de México (UNAM), C.P. 04510, México, D.F., Mexico.

Efimov D. is also with Department of Control Systems and Informatics, Saint Petersburg State University of Information Technologies Mechanics and Optics (ITMO), 49 Kronverkskiy av., 197101 Saint Petersburg, Russia.

Perruquetti W. is also with LAGIS (UMR-CNRS 8146), Ecole Centrale de Lille, BP 48, Cité Scientifique, 59651 Villeneuve-d'Ascq, France. interesting to apply this concept for time-varying systems. An extension of the homogeneity concept to time-varying systems has been given in [17], [18], where in the latter a reparametrization of time has also been required together with the state dilation. In this work we will apply the weighted homogeneity theory for the system dynamics considering the time variable as a constant parameter (it slightly differs from [18] and is similar to [17]).

Establishing stability properties, it is also important to quantify the rate of convergence in the system: exponential, asymptotic, finite-time or fixed-time [19], [20], [11], [21], [22]. Frequently, the homogeneity theory is used to establish finite-time or fixed-time stability [8], [2], [23], [22]: for example, if a system is globally asymptotically stable and homogeneous of negative degree, then it is finite-time stable.

The outline of this work is as follows. The preliminary definitions and the homogeneity framework are given in Section 2. The property of scaling of solutions for some class of homogeneous time-varying systems is presented in Section 3. Application of the developed theory to the problem of stability and convergence analysis for an adaptive estimator is considered in Section 4.

\section{Preliminaries}

Consider a time-varying differential equation [24]:

$$
d x(t) / d t=f(t, x(t)), t \geq t_{0}, t_{0} \in \mathbb{R},
$$

where $x(t) \in \mathbb{R}^{n}$ is the state vector; $f: \mathbb{R}^{n+1} \rightarrow \mathbb{R}^{n}$ is a continuous function with respect to $x$ and piecewise continuous with respect to $t, f(t, 0)=0$. We assume that solution of the system (1) for an initial condition $x_{0} \in \mathbb{R}^{n}$ at time instant $t_{0} \in \mathbb{R}$ is denoted as $x\left(t, t_{0}, x_{0}\right)$ and it is defined on some finite time interval $\left[t_{0}, t_{0}+T\right.$ ) (we will use the notation $x(t)$ to reference $x\left(t, t_{0}, x_{0}\right)$ if the origin of $x_{0}$ and $t_{0}$ is clear from the context).

A continuous function $\sigma: \mathbb{R}_{+} \rightarrow \mathbb{R}_{+}$belongs to class $\mathcal{K}$ if it is strictly increasing and $\sigma(0)=0$; it belongs to class $\mathcal{K}_{\infty}$ if it is also radially unbounded. A continuous function $\beta: \mathbb{R}_{+} \times \mathbb{R}_{+} \rightarrow \mathbb{R}_{+}$belongs to class $\mathcal{K} \mathcal{L}$ if $\beta(\cdot, r) \in \mathcal{K}$ and $\beta(r, \cdot)$ is a strictly decreasing to zero for any fixed $r \in \mathbb{R}_{+}$. Denote a sequence of integers $1, \ldots, m$ as $\overline{1, m}$.

\section{A. Stability definitions}

Let $\Omega$ be an open subset of $\mathbb{R}^{n}, 0 \in \Omega$. 
Definition 1. [24] At the steady state $x=0$ the system (1) is said to be

(a) uniformly stable if for any $\epsilon>0$ there is $\delta(\epsilon)$ such that for any $x_{0} \in \Omega$, if $\left|x_{0}\right| \leq \delta(\epsilon)$ then $\left|x\left(t, t_{0}, x_{0}\right)\right| \leq \epsilon$ for all $t \geq t_{0}$ for any $t_{0} \in \mathbb{R}$;

(b) uniformly asymptotically stable if it is uniformly stable and for any $\kappa>0$ and $\epsilon>0$ there exists $T(\kappa, \epsilon) \geq 0$ such that for any $x_{0} \in \Omega$, if $\left|x_{0}\right| \leq \kappa$ then $\left|x\left(t, t_{0}, x_{0}\right)\right| \leq \epsilon$ for all $t \geq t_{0}+T(\kappa, \epsilon)$ for any $t_{0} \in \mathbb{R}$;

If $\Omega=\mathbb{R}^{n}$, then the corresponding properties are called global uniform stability/asymptotic stability of $x=0$.

Proposition 1. [24] The steady state $x=0$ of the system (1) is

- uniformly stable iff there is a function $\sigma \in \mathcal{K}_{\infty}$ such that $\left|x\left(t, t_{0}, x_{0}\right)\right| \leq \sigma\left(\left|x_{0}\right|\right)$ for all $t \geq t_{0}$, any $t_{0} \in \mathbb{R}$ and all $x_{0} \in \Omega$;

- uniformly asymptotically stable iff there is a function $\beta \in$ $\mathcal{K} \mathcal{L}$ such that $\left|x\left(t, t_{0}, x_{0}\right)\right| \leq \beta\left(\left|x_{0}\right|, t-t_{0}\right)$ for all $t \geq t_{0}$, any $t_{0} \in \mathbb{R}$ and all $x_{0} \in \Omega$.

\section{B. Homogeneity}

For any $r_{i}>0, i=\overline{1, n}$ and $\lambda>0$, define the dilation matrix $\Lambda_{r}(\lambda)=\operatorname{diag}\left\{\lambda^{r_{i}}\right\}_{i=1}^{n}$ and the vector of weights $r=$ $\left[r_{1}, \ldots, r_{n}\right]^{T}$.

For any $r_{i}>0, i=\overline{1, n}$ and $x \in \mathbb{R}^{n}$ the homogeneous norm can be defined as follows

$$
|x|_{r}=\left(\sum_{i=1}^{n}\left|x_{i}\right|^{\rho / r_{i}}\right)^{1 / \rho}, \rho=\prod_{i=1}^{n} r_{i} .
$$

For all $x \in \mathbb{R}^{n}$, its Euclidean norm $|x|$ is related with the homogeneous one:

$$
\underline{\sigma}_{r}\left(|x|_{r}\right) \leq|x| \leq \bar{\sigma}_{r}\left(|x|_{r}\right)
$$

for some $\underline{\sigma}_{r}, \bar{\sigma}_{r} \in \mathcal{K}_{\infty}$. In the following, due to this "equivalence", stability analysis with respect to the norm $|x|$ will be substituted with analysis for the norm $|x|_{r}$. The homogeneous norm has an important property that is $\left|\Lambda_{r}(\lambda) x\right|_{r}=\lambda|x|_{r}$ for all $x \in \mathbb{R}^{n}$. Define $\mathbb{S}_{r}=\left\{x \in \mathbb{R}^{n}:|x|_{r}=1\right\}$.

Definition 2. [1] The function $g: \mathbb{R}^{n} \rightarrow \mathbb{R}$ is called $r$ homogeneous $\left(r_{i}>0, i=\overline{1, n}\right)$, if for any $x \in \mathbb{R}^{n}$ the relation

$$
g\left(\Lambda_{r}(\lambda) x\right)=\lambda^{d} g(x)
$$

holds for some $d \in \mathbb{R}$ and all $\lambda>0$.

The function $f: \mathbb{R}^{n} \rightarrow \mathbb{R}^{n}$ is called $r$-homogeneous $\left(r_{i}>\right.$ $0, i=\overline{1, n})$, if for any $x \in \mathbb{R}^{n}$ the relation

$$
f\left(\Lambda_{r}(\lambda) x\right)=\lambda^{d} \Lambda_{r}(\lambda) f(x)
$$

holds for some $d \geq-\min _{1 \leq i \leq n} r_{i}$ and all $\lambda>0$.

In both cases, the constant $d$ is called the degree of homogeneity.

A dynamical system

$$
\dot{x}(t)=f(x(t)), t \geq 0
$$

is called $r$-homogeneous of degree $d$ if this property is satisfied for the vector function $f$ in the sense of Definition 2. An advantage of homogeneous systems described by nonlinear ordinary differential equations is that any of its solution can be obtained from another solution under the dilation rescaling and a suitable time re-parametrization:

Proposition 2. Let $x: \mathbb{R}_{+} \rightarrow \mathbb{R}^{n}$ be a solution of the $r$ homogeneous system (2) with the degree $d$ for an initial condition $x_{0} \in \mathbb{R}^{n}$. For any $\lambda>0$ define $y(t)=\Lambda_{r}(\lambda) x\left(\lambda^{d} t\right)$ for all $t \geq 0$, then $y(t)$ is also a solution of (2) with the initial condition $y_{0}=\Lambda_{r}(\lambda) x_{0}$.

In order to apply the weighted homogeneity property, introduced for time-invariant systems in Definition 2, to the time-varying systems (1) we need an extended concept.

Definition 3. [17] The function $g: \mathbb{R}^{n+1} \rightarrow \mathbb{R}$ is called $r$-homogeneous $\left(r_{i}>0, i=\overline{1, n}\right)$, if for any $x \in \mathbb{R}^{n}$ and $t \in \mathbb{R}$ the relation

$$
g\left(t, \Lambda_{r}(\lambda) x\right)=\lambda^{d} g(t, x)
$$

holds for some $d \in \mathbb{R}$ and all $\lambda>0$.

The function $f: \mathbb{R}^{n+1} \rightarrow \mathbb{R}^{n}$ is called $r$-homogeneous $\left(r_{i}>0, i=\overline{1, n}\right)$, if for any $x \in \mathbb{R}^{n}$ and $t \in \mathbb{R}$ the relation

$$
f\left(t, \Lambda_{r}(\lambda) x\right)=\lambda^{d} \Lambda_{r}(\lambda) f(t, x)
$$

holds for some $d \geq-\min _{1 \leq i \leq n} r_{i}$ and all $\lambda>0$.

Thus in the time-varying case (1) the homogeneity will be verified considering $t$ as a constant parameter.

\section{MAIN RESUlTS}

This section has three parts. First, an extension of Proposition 2 is presented for time-varying system (1), and some useful tools for uniform stability analysis of nonlinear timevarying systems are introduced. Second, relation with time scaling is analyzed.

\section{A. Scaling solutions of homogeneous time-varying systems}

We will consider the following modification of the system (1):

$$
d x(t) / d t=f(\omega t, x(t)), t \geq t_{0}, t_{0} \in \mathbb{R},
$$

for some $\omega>0$. The parameter $\omega$ represents dependence on the convergence rate of time processes in the system or the frequency of time-varying part. For an initial condition $x_{0} \in \mathbb{R}^{n}$ at initial time $t_{0}$ denote the corresponding solution of (3) as $x_{\omega}\left(t, t_{0}, x_{0}\right)$, thus $x\left(t, t_{0}, x_{0}\right)=x_{1}\left(t, t_{0}, x_{0}\right)$. We have in this case the following extension of Proposition 2 (a variant of this result has been formulated in the proof of Theorem 2 in [17]).

Proposition 3. Let $x\left(t, t_{0}, x_{0}\right)$ be a solution of the $r$ homogeneous system (1) with the degree $d$ for an initial condition $x_{0} \in \mathbb{R}^{n}$ and $t_{0} \in \mathbb{R}$. For any $\lambda>0$ the system (3) with $\omega=\lambda^{d}$ has a solution $y\left(t, t_{0}, y_{0}\right)=$ 
$\Lambda_{r}(\lambda) x\left(\lambda^{d} t, \lambda^{d} t_{0}, x_{0}\right)$ for all $t \geq t_{0}$ with the initial condition $y_{0}=\Lambda_{r}(\lambda) x_{0}$.

All the proofs are omitted due to space limitations. For the case $d=0$ we recover that all solutions of (1) are interrelated as in Proposition 2 (as in time-invariant case). It is well known fact that for the ordinary differential equation (2) local attractiveness implies global asymptotic stability [1]. In the present setting that result has the following correspondence (a similar conclusion also can be found in the proof of Theorem 2 in [17]).

Lemma 1. Let the system (1) be r-homogeneous with degree $d \neq 0$ and globally uniformly asymptotically stable, i.e. there is $\beta \in \mathcal{K} \mathcal{L}$ such that

$$
\left|x\left(t, t_{0}, x_{0}\right)\right|_{r} \leq \beta\left(\left|x_{0}\right|_{r}, t-t_{0}\right) \quad \forall t \geq t_{0}
$$

for any $x_{0} \in \mathbb{R}^{n}$ and any $t_{0} \in \mathbb{R}$. Then (3) is globally uniformly asymptotically stable for any $\omega>0$ and

$$
\left|x_{\omega}\left(t, t_{0}, x_{0}\right)\right|_{r} \leq \beta_{\omega}\left(\left|x_{0}\right|_{r}, t-t_{0}\right) \quad \forall t \geq t_{0}
$$

for any $x_{0} \in \mathbb{R}^{n}$ and any $t_{0} \in \mathbb{R}$, where $\beta_{\omega}(s, t)=$ $\omega^{1 / d} \beta\left(\omega^{-1 / d} s, \omega t\right)$.

It is a well known fact for linear time-varying systems (homogeneous systems of degree $d=0$ ) that its stability for some $\omega$ does not imply stability for all $\omega \in(0,+\infty)$. For nonlinear homogeneous time-varying systems with degree $d \neq 0$ this is not the case, according to the result of Lemma 1 if they are globally uniformly stable for some $\omega$, then they preserve the uniform stability for an arbitrary $\omega>0$. This is an intriguing advantage of "nonlinear" time-varying systems. In addition, it is shown in Lemma 1 that the rate of convergence will be scaled by $\omega$, thus the time of transients in these systems is predefined by the time-varying part, which is not the case for the degree $d=0$, where the rate of convergence cannot be modified by $\omega$ (see Proposition 3 )!

Further let us consider several useful consequences of Proposition 3 and Lemma 1.

Corollary 1. Let the system (1) be r-homogeneous with degree $d=0$ and uniformly asymptotically stable into the set $\Omega=B_{\rho}=\left\{x \in \mathbb{R}^{n}:|x|_{r} \leq \rho\right\}$ for some $0<\rho<+\infty$, then (1) is globally uniformly asymptotically stable.

Corollary 2. Let the system (3) be r-homogeneous with degree $d \neq 0$ and uniformly asymptotically stable into the set $\Omega=B_{\rho}=\left\{x \in \mathbb{R}^{n}:|x|_{r} \leq \rho\right\}$ for a fixed $0<\rho<+\infty$ for any $\omega>0$, then (3) is globally uniformly asymptotically stable for any $\omega>0$.

Corollary 3. Let the system (3) be r-homogeneous with degree $d>0$ and uniformly asymptotically stable into the set $B_{\rho}$ for some $0<\rho<+\infty$ for any $0 \leq \omega \leq \omega_{0}$ with $0<\omega_{0}<+\infty$, then (3) is globally uniformly asymptotically stable for any $\omega>0$.

Corollary 4. Let the system (3) be r-homogeneous with degree $d<0$ and uniformly asymptotically stable with respect to the set $B_{\rho}$ for some $0<\rho<+\infty$ for any $0 \leq \omega \leq \omega_{0}$ with $0<\omega_{0}<+\infty^{1}$, then (3) is globally uniformly asymptotically stable for any $\omega>0$.

The results presented in this section open a possibility for stability analysis of nonlinear time-varying systems. In particular, they can be interpreted as robustness of the timeinvariant homogeneous systems with respect to time-varying perturbations of parameters, i.e. if for one value of parameter $\omega$ for this time-varying perturbation the system is stable, then it can be stable for any other value of $\omega$.

\section{B. Robustness with respect to time scaling}

Consider for some $a>0$ the following modification of (1) or (3)

$$
d z(t) / d t=a f(a t, z(t)), t \geq t_{0}, t_{0} \in \mathbb{R},
$$

where $z \in \mathbb{R}^{n}$ is the state function. Then $z\left(t, t_{0}, x_{0}\right)=$ $x\left(a t, a t_{0}, x_{0}\right)$ for $t \geq t_{0}$ is a solution of (4) for the initial condition $x_{0} \in \mathbb{R}^{n}$. Indeed,

$$
\begin{gathered}
d z\left(t, t_{0}, x_{0}\right) / d t=d x\left(a t, a t_{0}, x_{0}\right) / d t=a d x\left(a t, a t_{0}, x_{0}\right) / d a t \\
=a f\left[a t, x\left(a t, a t_{0}, x_{0}\right)\right]=a f\left(a t, z\left(t, t_{0}, x_{0}\right)\right) .
\end{gathered}
$$

Thus time scaling (multiplication on $a$ ) acts similarly on solutions as the dilation transformation in homogeneous systems, and the following conclusion on the system stability can be obtained.

Lemma 2. Let the system (1) be globally uniformly asymptotically stable, then (4) is globally uniformly asymptotically stable for any $a>0$, and the rate of convergence in (4) is scaled by a with respect to (1).

Consequently, for a homogeneous system (1) we may obtain an extension of Proposition 3.

Proposition 4. Let $x\left(t, t_{0}, x_{0}\right)$ be a solution of the $r$ homogeneous system (1) with the degree $d$ for an initial condition $x_{0} \in \mathbb{R}^{n}$ and $t_{0} \in \mathbb{R}$. For any $\lambda>0$ and $a>0$ the system

$$
d w(t) / d t=a f(a \omega t, w(t)), t \geq t_{0}, t_{0} \in \mathbb{R},
$$

with $\omega=\lambda^{d}$ has a solution $w\left(t, t_{0}, w_{0}\right)=$ $\Lambda_{r}(\lambda) x\left(a \lambda^{d} t, a \lambda^{d} t_{0}, x_{0}\right)$ for all $t \geq t_{0}$ with the initial condition $w_{0}=\Lambda_{r}(\lambda) x_{0}$.

Therefore, for $a=\lambda^{-d}$ the systems (1) and (5) have the same rates of convergence, and their corresponding solutions differ in amplitudes only.

\section{APPLICATION TO ADAPTIVE ESTIMATION}

In this section the previously proposed results will be applied to analyze the convergence of the error dynamics given by a nonlinear estimation algorithm.

\footnotetext{
${ }^{1}$ In this case for each $0<\omega<\omega_{0}$, any $\varepsilon>0$ and $\kappa>0$ there is $0 \leq T_{\kappa, \varepsilon}^{\omega}<+\infty$ such that $\left|x_{\omega}\left(t, \bar{t}_{0}, x_{0}\right)\right|_{r} \leq \rho+\varepsilon$ for all $t \geq t_{0}+T_{\kappa, \varepsilon}^{\omega}$ for any $x_{0} \in B_{\kappa}$, and $\left|x\left(t, t_{0}, x_{0}\right)\right|_{r} \leq \sigma_{\omega}\left(\left|x_{0}\right|_{r}\right)$ for all $t \geq t_{0}$ for some function $\sigma_{\omega} \in \mathcal{K}$ for all $t_{0} \in \mathbb{R}$.
} 


\section{A. Problem statement}

Consider the following time-varying system

$$
\frac{d x(t)}{d t}=\Gamma^{T}(t) \theta
$$

where $\Gamma: \mathbb{R} \rightarrow \mathbb{R}^{q}$ is a continuous function of time, and $\theta \in$ $\mathbb{R}^{q}$. The term $\Gamma(t)$ is known as the regressor vector, satisfying the well-known persistence of excitation condition [25], and $\theta$ is the unknown parameter vector. It is assumed that $x(t)$ is available for measurements, and in order to estimate the vector $\theta$ the following nonlinear algorithm can be introduced

$$
\begin{aligned}
& \dot{\hat{x}}(t)=-k_{1}\lceil\hat{x}(t)-x(t)\rfloor^{\gamma}+\Gamma^{T}(t) \hat{\theta}(t), \quad \hat{x}(0)=0, \\
& \dot{\hat{\theta}}(t)=-k_{2}\lceil\hat{x}(t)-x(t)\rfloor^{2 \gamma-1} \Gamma(t), \quad \hat{\theta}(0)=[0 \ldots 0]^{T},
\end{aligned}
$$

where $\lceil\cdot\rfloor^{\gamma} \doteq|\cdot|^{\gamma} \operatorname{sign}(\cdot), k_{1}, k_{2}>0$ are positive gains, and the parameter $\gamma \in(0.5,1)$ (for the case $\gamma=1$ the estimator reduces to the well known linear adaptive observer [25]). Let us define the errors $\tilde{x}(t)=\hat{x}(t)-x(t)$ and $\tilde{\theta}(t)=\hat{\theta}(t)-\theta$. Hence, the error dynamics is given by

$$
\begin{aligned}
\dot{\tilde{x}}(t) & =-k_{1}\lceil\tilde{x}(t)\rfloor^{\gamma}+\Gamma^{T}(t) \tilde{\theta}(t), \\
\dot{\tilde{\theta}}(t) & =-k_{2}\lceil\tilde{x}(t)\rfloor^{2 \gamma-1} \Gamma(t) .
\end{aligned}
$$

It is clear that the system (6)-(7) has the form (1), and also it is possible to find a modification to express it in the form (3):

$$
\begin{aligned}
& \dot{\tilde{x}}(t)=-k_{1}\lceil\tilde{x}(t)\rfloor^{\gamma}+\Gamma^{T}(\omega t) \tilde{\theta}(t), \\
& \dot{\tilde{\theta}}(t)=-k_{2}\lceil\tilde{x}(t)\rfloor^{2 \gamma-1} \Gamma(\omega t) .
\end{aligned}
$$

System (6)-(7) is $r$-homogeneous with degree $d=\gamma-1$ for $\left(r_{1}, r_{2}, \ldots, r_{q+1}\right)=(1, \gamma, \ldots, \gamma)$. Note that $d<0$ for all $\gamma \in(0.5,1)$. It is possible to demonstrate that system (6)-(7) is asymptotically stable. We omit the proof of this fact because it is beyond the scope of this paper. However, the proof is based on the utilization of the Lyapunov function $V(\tilde{x}, \tilde{\theta})=\frac{1}{2 \gamma} \tilde{x}^{2 \gamma}+\frac{1}{2 k_{2}} \tilde{\theta}^{T} \tilde{\theta}$ and the invariance principle for time-varying systems from [26]. Since (6)-(7) is homogeneous, the rate of convergence for its modified version (8)-(9) can be evaluated using Lemma 1 from the convergence of the original system (6)-(7). The modification (8)-(9) corresponds to a frequency change in the regressor vector, that is a usual instrument in the adaptive estimation. According to the results of Proposition 3 and Lemma 1, for the linear estimator with $\gamma=1$ the speed of error convergence should not be modified, while for the nonlinear observer (8)-(9) with $\gamma \in(0.5,1)$ the frequency $\omega$ may impact the convergence (note that we do not know the form of the function $\beta \in \mathcal{K} \mathcal{L}$ from Lemma 1, in general rescaling by $\omega^{-1 / d}$ of the initial conditions may cancel the rate improvement by $\omega t$ ).

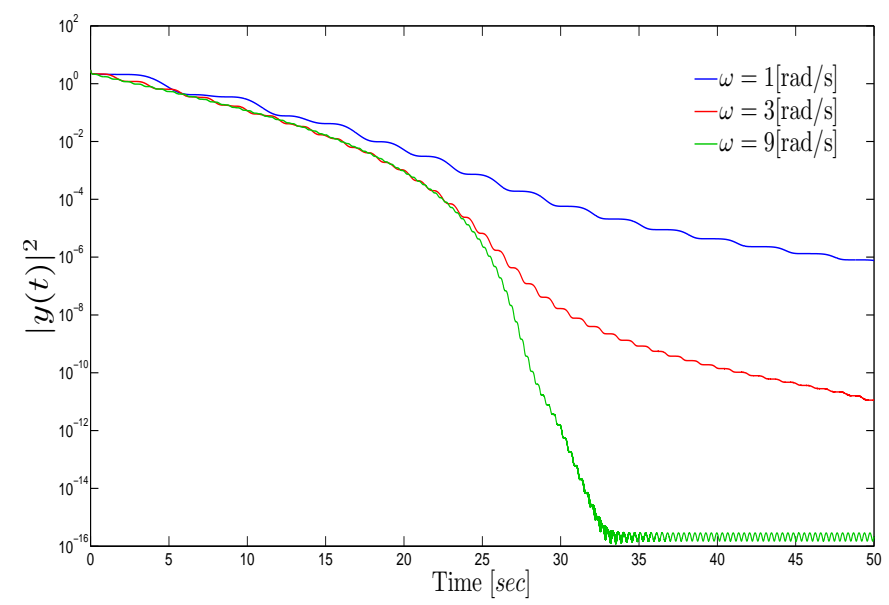

Figure 1. Estimation Error Convergence for Nonlinear Adaptive Observer $(\gamma=0.75), y(t)=\left[\begin{array}{cc}\tilde{x}(t) & \tilde{\theta}^{T}(t)\end{array}\right]^{T}$.

\section{B. Example}

Let us illustrate such a convergence improvement for the example:

$$
\frac{d x(t)}{d t}=\sin \omega t-1=\Gamma^{T}(t) \theta, \quad x(0)=1,
$$

where $\Gamma(t)=\left[\begin{array}{ll}\sin \omega t & 1\end{array}\right]^{T}$ and $\theta=\left[\begin{array}{ll}1 & -1\end{array}\right]^{T}$. Let us consider the case $\gamma=0.75$ with $k_{1}=10, k_{2}=0.1$. Some simulations, for different values of $\omega$, have been done in MatLab Simulink environment, with Euler discretization method and sampling time equal to $0.01[\mathrm{sec}]$. The simulation results, confirming Lemma 1 statements, are presented in Figs. 1-2 in comparison with the linear estimation algorithm for $\gamma=1$. The square norm of estimation error convergence for nonlinear and linear adaptive observers are shown in logarithmic scale in Fig. 1 and Fig. 2, respectively. As we can conclude, increasing the frequency we can improve the rate of convergence for the nonlinear algorithm in certain limits, while for the linear algorithm there is no significant improvement.

Therefore, application of the nonlinear homogeneous algorithms may serve for improvement of the rate of estimation depending on the available excitation frequency of the timevarying regressor.

\section{CONCLusions}

The homogeneity theory extensions are obtained for timevarying systems. It is shown that for any degree of homogeneity the solutions of a homogeneous system are interrelated subject to the time rescaling. Next, this fact is utilized in order to show that local uniform asymptotic stability of homogeneous systems implies global one, and that for nonlinear homogeneous systems with non-zero degree global asymptotic stability for a parameter endorses this property for an arbitrary value of this parameter. The possibility of finite-time stability in time-varying systems is a future work. 


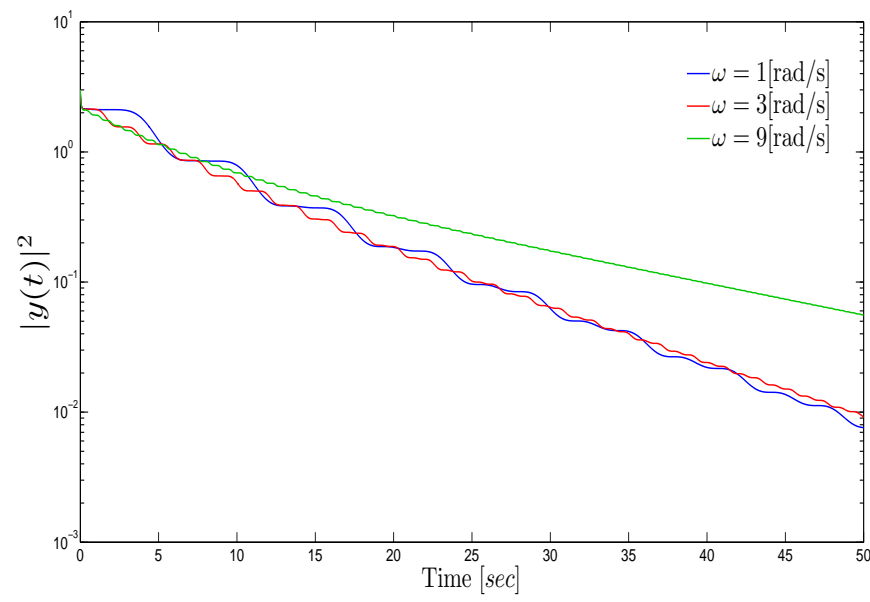

Figure 2. Estimation Error Convergence for Linear Adaptive Observer $(\gamma=$ 1), $y(t)=\left[\begin{array}{cc}\tilde{x}(t) & \tilde{\theta}^{T}(t)\end{array}\right]^{T}$.

Efficiency of the proposed approach is demonstrated for an adaptive estimation problem benchmark. Application of the developed results for analysis and design of control or estimation algorithms in time-varying systems is a direction of future research.

\section{ACKNOWLEDGMENT}

This work was supported in part by the Government of Russian Federation (Grant 074-U01) and the Ministry of Education and Science of Russian Federation (Project 14.Z50.31.0031).

\section{REFERENCES}

[1] A. Bacciotti and L. Rosier, Liapunov Functions and Stability in Control Theory, vol. 267 of Lecture Notes in Control and Inform. Sci. Berlin: Springer, 2001.

[2] V. Andrieu, L. Praly, and A. Astolfi, "Homogeneous approximation, recursive observer design, and output feedback," SIAM J. Control Optimization, vol. 47, no. 4, pp. 1814-1850, 2008.

[3] H. Hermes, Differential Equations: Stability and Control, vol. 109 of Lecture Notes in Pure Appl. Math., ch. Homogeneous coordinates and continuous asymptotically stabilizing feedback controls, pp. 249-260. Marcel Dekker, 1991

[4] Y. Hong, "Finite-time stabilization and stabilizability of a class of controllable systems," Systems\&Control Lett., vol. 46, pp. 231-236, 2002.

[5] L. Rosier, "Homogeneous Lyapunov function for homogeneous continuous vector field," Systems\&Control Lett., vol. 19, pp. 467-473, 1992.

[6] H. Hermes, "Nilpotent and high-order approximations of vector field systems," SIAM Review, vol. 33, no. 2, pp. 238-264, 1991.

[7] T. Ménard, E. Moulay, and W. Perruquetti, "Homogeneous approximations and local observer design," ESAIM: Control, Optimization and Calculus of Variations, vol. 19, no. 3, pp. 906-929, 2013.

[8] S. Bhat and D. Bernstein, "Geometric homogeneity with applications to finite-time stability," Mathematics of Control, Signals and Systems, vol. 17, pp. 101-127, 2005.

[9] L. Grüne, "Homogeneous state feedback stabilization of homogeneous systems," SIAM J. Control Optimization, vol. 38, no. 4, pp. 1288-1314, 2000.

[10] M. Kawski, Homogeneous feedback stabilization, vol. 7 of Progress in systems and control theory: New trends in systems theory. Birkhäuser, 1991.
[11] E. Moulay and W. Perruquetti, "Finite time stability and stabilization of a class of continuous systems," J. Mathematical Analysis Applications, vol. 323, no. 2, pp. 1430-1443, 2006.

[12] R. Sepulchre and D. Aeyels, "Stabilizability does not imply homogeneous stabilizability for controllable systems," SIAM J. Control Optimization, vol. 34, no. 5, pp. 1798-1813, 1996.

[13] V. Zubov, "On systems of ordinary differential equations with generalized homogenous right-hand sides," Izvestia vuzov. Mathematica. vol. 1, pp. 80-88, 1958. in Russian.

[14] D. Efimov and W. Perruquetti, "Oscillations conditions in homogenous systems," in Proc. NOLCOS'10, (Bologna), pp. 1379-1384, 2010.

[15] E. Bernuau, A. Polyakov, D. Efimov, and W. Perruquetti, "On iss and iiss properties of homogeneous systems," in Proc. European Control Conference (ECC) 2013, (Zürich), 2013.

[16] E. Bernuau, A. Polyakov, D. Efimov, and W. Perruquetti, "Verification of iss, iiss and ioss properties applying weighted homogeneity," Systems \& Control Letters, vol. 62, no. 12, pp. 1159-1167, 2013.

[17] J. Peuteman and D. Aeyels, "Averafing results and the study of uniform asymptotic stability of homogeneous differential equations that are not fast time-varying," SIAM J. Control Optim., vol. 37, no. 4, pp. 9971010, 1999.

[18] Y. Orlov, "Finite time stability and robust control synthesis of uncertain switched systems," SIAM J. Control Optim., vol. 43, no. 4, pp. $1253-$ $1271,2005$.

[19] E. Roxin, "On finite stability in control systems," Rendiconti del Circolo Matematico di Palermo, vol. 15, pp. 273-283, 1966.

[20] P. Dorato, "An overview of finite-time stability," in Current Trends in Nonlinear Systems and Control (L. Menini, L. Zaccarian, and C. T. Abdallah, eds.), Systems \& Control: Foundations \& Applications, pp. 185-194, Boston: Birkhäuser, 2006.

[21] S. Nersesov, W. Haddad, and Q. Hui, "Finite-time stabilization of nonlinear dynamical systems via control vector lyapunov functions," J. Franklin Inst., vol. 345, pp. 819-837, 2008.

[22] A. Polyakov, "Nonlinear feedback design for fixed-time stabilization of linear control systems," Automatic Control, IEEE Transactions on, vol. 57, no. 8, pp. 2106-2110, 2012.

[23] E. Cruz-Zavala, J. Moreno, and L. Fridman, "Uniform robust exact differentiator," IEEE Trans. Automat. Control, vol. 56, no. 11, pp. $2727-$ 2733, 2011.

[24] H. Khalil, Nonlinear Systems. Upper Saddle River, New Jersey: Prentice Hall, 3rd ed., 2002.

[25] K. Narendra and A. Annaswamy, Stable Adaptive Systems. Mineola, NY: Dover Publications, 2005

[26] D. Aeyels, "Asymptotic stability of nonautonomous systems by liapunov's direct method," Systems \& Control Letters, vol. 25, pp. 273 $280,1995$. 